1999-05-01

\title{
The Mycobactericidal Efficacy of Orthophthalaldehyde and the Comparative Resistances of Mycobacterium Bovis, Mycobacterium Terrae, and Mycobacterium Chelonae
}

\author{
Richard A. Robison \\ richard_robison@byu.edu \\ Adam W. Gregory \\ G. Bruce Schaalje \\ Jonathan D. Smart
}

Follow this and additional works at: https://scholarsarchive.byu.edu/facpub

Part of the Microbiology Commons

\author{
Original Publication Citation \\ Infection Control and Hospital Epidemiology 2.5(May 1999): 324-33.
}

\section{BYU ScholarsArchive Citation}

Robison, Richard A.; Gregory, Adam W.; Schaalje, G. Bruce; and Smart, Jonathan D., "The Mycobactericidal Efficacy of Orthophthalaldehyde and the Comparative Resistances of Mycobacterium Bovis, Mycobacterium Terrae, and Mycobacterium Chelonae" (1999). Faculty Publications. 620.

https://scholarsarchive.byu.edu/facpub/620

This Peer-Reviewed Article is brought to you for free and open access by BYU ScholarsArchive. It has been accepted for inclusion in Faculty Publications by an authorized administrator of BYU ScholarsArchive. For more information, please contact ellen_amatangelo@byu.edu. 


\title{
The Mycobactericidal Efficacy of ORTHO- Phthalaldehyde and the Comparative Resistances OF MYCOBACTERIUM BOVIS, MYCOBACTERIUM TERRAE, AND MYCOBACTERIUM CHELONAE
}

\author{
Adam W. Gregory, BS; G. Bruce Schaalje, PhD; Jonathan D. Smart, BS; Richard A. Robison, PhD
}

\begin{abstract}
OBJECTIVES: To assess the mycobactericidal efficacy of an agent relatively new to disinfection, ortho-phthalaldehyde (OPA) and to compare the resistances of three Mycobacterium species. Mycobacterium bovis (strain BCG) was compared with Mycobacterium chelonae and Mycobacterium terrae to investigate the feasibility of using either of the latter two species in tuberculocidal testing. $M$ chelonae (a rapid grower) and $M$ terrae (an intermediate grower) both grow faster and are less virulent than $M$ bovis (a slow grower).

DESIGN: The quantitative suspension protocol specified by the Environmental Protection Agency (EPA), the Tuberculocidal Activity Test Method (EPA test), was used throughout this study. Standard suspensions of all three species were prepared in a similar manner. Two suspensions of $M$ bovis, created in different laboratories, were used. These were tested against two concentrations of alkaline glutaraldehyde to provide reference data. Two concentrations of OPA were evaluated
\end{abstract}

against all mycobacterial test suspensions. Four replicates of each organism-disinfectant combination were performed.

RESULTS: Results were assessed by analysis of variance. $M$ terrae was significantly more resistant to $0.05 \%$ OPA than either $M$ bovis or $M$ chelonae. At $0.21 \%$ OPA, $M$ terrae was slightly more susceptible than one test suspension of $M$ bovis, but not significantly different from the other. M chelonae was significantly less resistant than the other species at both OPA concentrations. At their respective minimum effective concentration, OPA achieved a $6-\log _{10}$ reduction of $M$ bovis in nearly one sixth the time required by glutaraldehyde ( 5.5 minutes vs 32 minutes).

CONCLUSIONS: These data, along with other recent studies, lend support to the idea that $M$ terrae may be a suitable test organism for use in the tuberculocidal efficacy testing of disinfectants. They also confirm the relatively rapid tuberculocidal activity of OPA (Infect Control Hosp Epidemiol 1999;20:324-330).
While airborne transmission of tuberculosis (TB) is the most common route of spread, cases due to indirect contact with semicritical medical instruments (such as bronchoscopes and endoscopes) continue to occur. ${ }^{1-4}$ Contributing to this problem are exaggerated tuberculocidal label claims, which have been discovered even on highlevel disinfectants. ${ }^{5-7}$ Worse yet, some disinfectants have been found to support the presence of contaminating bacteria for extended periods of time..$^{8-10}$ Because mycobacteria generally are more resistant to chemical disinfection than most other vegetative organisms, ${ }^{11}$ these data raise special concerns with respect to Mycobacterium tuberculosis. One way to reduce the incidence of disease caused by inadequately disinfected medical devices is to assure that tuberculocidal assessment of disinfectants is accomplished with accurate and standardized test procedures.
One of the most critical variables in tuberculocidal testing is the selection of the challenge organism (which should possess resistance equivalent to that of $M$ tuberculosis). This has been a source of variability in past studies, contributing to the difficulty of establishing a truly standardized protocol. The quantitative suspension procedure currently approved by the Environmental Protection Agency (EPA) was described first in 1987 by Ascenzi et al. ${ }^{5}$ Known as the EPA Tuberculocidal Activity Test Method (EPA test), this quantitative procedure has attempted to control many of the variables involved in tuberculocidal testing. It specifies the use of Mycobacterium bovis (strain $\mathrm{BCG}$ ) as the challenge organism. ${ }^{12} \mathrm{M}$ bovis also is specified in the Association of Official Analytical Chemists tuberculocidal procedure. ${ }^{13}$ It is related closely to $M$ tuberculosis and has demonstrated similar levels of resistance to a vari-

From the Department of Microbiology (Dr. Robison, Mr. Gregory, and Mr. Smart); the Department of Statistics (Dr. Schaalje), Brigham Young University, Provo, Utah.

This work was supported by a grant from the Professional Development Committee of Brigham Young University and Johnson \& Johnson Medical, Inc. The authors thank Heather Hall-Albert, Melissa Hardy, Jess Price, Jonathan Olivier, and Sara Maycock-Olivier for their many hours of generous technical assistance.

Address reprint requests to Richard A. Robison, PhD, 791 WIDB, Department of Microbiology, Brigham Young University, Provo, UT 84602-5133.

98-OA-057. Gregory AW, Schaalje GB, Smart JD, Robison RA. The mycobactericidal efficacy of ortho-phthalaldehyde and the comparative resistances of Mycobacterium bovis, Mycobacterium terrae, and Mycobacterium chelonae. Infect Control Hosp Epidemiol 1999;20:324-330. 
ety of disinfecting agents. ${ }^{14}$ However, the use of $M$ bovis has drawbacks. In addition to being more virulent than several other known strains, it is slow-growing, requiring at least 3 weeks before final results can be obtained.

Several researchers have experimented with fastergrowing, less pathogenic strains of mycobacteria in hopes of identifying a more suitable test species. This study involves a comparative analysis of a rapid-growing species (Mycobacterium chelonae) and an intermediate-growing species (Mycobacterium terrae) with $M$ bovis (a slow-growing species).

The comparative resistances of these strains were evaluated using ortho-phthalaldehyde (OPA), a compound relatively new to disinfection. The current standard for high-level disinfection of semicritical instruments is $2 \%$ alkaline glutaraldehyde. Although this agent has well-documented bactericidal, sporicidal, and mycobactericidal activity, it is not without deficiencies, including inactivation by spontaneous polymerization and loss of activity with dilution. ${ }^{15,16}$ It also requires a relatively long exposure time to exact a significant tuberculosis kill. ${ }^{17}$

Because OPA is a relatively novel disinfectant, few efficacy studies have been performed. However, the limited available data suggest that it has much higher activity than $2 \%$ glutaraldehyde, especially with respect to its tuberculocidal and virucidal action. In this study, we evaluated the mycobactericidal effects of two concentrations of OPA on the three mycobacterial species. For comparison purposes, data also were collected on two concentrations of glutaraldehyde using the current tuberculocidal test organism, $M$ bovis.

\section{METHODS}

\section{Test Organisms}

Stock suspensions of $M$ terrae (American Type Culture Collection [ATCC] 15755), $M$ chelonae (ATCC 35752 ), and $M$ bovis (ATCC 35743) were prepared according to the procedure specified in the EPA test protocol ${ }^{12}$ with the following modifications: The final scale-up was performed by adding $50 \mathrm{~mL}$ of culture to each of two 2-L plastic roller bottles containing $450 \mathrm{~mL}$ of modified ProskauerBeck medium, prepared in our laboratory, containing $0.1 \%$ Tween 80 . Cultures were incubated with loose caps at $37^{\circ} \mathrm{C}$, rolling slowly, for various times depending on the species.

As specified in the EPA protocol, when the absorbance of the culture at $500 \mathrm{~nm}$ reached a minimum of $0.6\left(1-5 \times 10^{8}\right.$ colony-forming units $\left.[\mathrm{CFU}] / \mathrm{mL}\right)$, the cells were harvested. On the day prior to harvest, Tween 80 was added to a final concentration of $0.1 \%$ in each bottle. The suspensions were homogenized in a Teflon-on-glass tissue grinder and frozen at $-70^{\circ} \mathrm{C}$, as specified.

\section{Disinfectants}

Four samples of disinfectant were used: two concentrations of alkaline gluteraldehyde (Cidex activated dialdehyde solution, Johnson \& Johnson Medical, Inc, Arlington, TX) and two concentrations of OPA (Cidex OPA solution, Johnson \& Johnson Medical, Inc). The two glutaraldehyde solutions came from the same lot. One was freshly activated
(2.5\%), and one was stressed through simulated reuse, as described below, and diluted to contain 1.5\% glutaraldehyde. This latter solution constitutes the manufacturer's minimum effective concentration. The OPA samples originated from a solution stressed through simulated use and diluted to contain $0.21 \%$ (the minimum effective concentration) and $0.05 \%$ OPA. The $0.05 \%$ OPA solution provided longer kill times, for more suitable comparison of the three Mycobacterium species. Simulated reuse of the disinfectant solutions consisted of the addition of $5 \%$ horse serum and the processing of two sets of anesthesia equipment for 42 cycles over 14 days, according to a standard reuse protocol. A $0.8 \%$ phenol standard, the control disinfectant solution specified in the EPA test method, also was assayed with each test suspension.

\section{Test Suspensions}

Mycobacterial test suspensions were prepared according to the EPA test protocol. On each test day, the required number of frozen stock vials was allowed to thaw. The contents of each vial were added to a prechilled $30-\mathrm{mL}$ Teflon-on-glass tissue homogenizer with an equal volume of phosphate-buffered gelatin. The mixture was homogenized on ice for 1 to 2 minutes. The homogenized suspension then was diluted using ice-cold physiological saline solution containing $0.1 \%$ Tween 80 to approximately $1 \times 10^{7}$ $\mathrm{CFU} / \mathrm{mL}$. This suspension was kept on ice until used to inoculate test disinfectants.

\section{Disinfectant Test Procedure}

All disinfectants were assayed according to the EPA test protocol. Each test consisted of inoculating the disinfectant at time zero with a standardized test organism suspension and sampling this mixture at predetermined time intervals. In all cases, tubes containing $9 \mathrm{~mL}$ of the appropriate test disinfectant were equilibrated in a $20^{\circ} \mathrm{C}$ water bath. At time zero, a 1-mL aliquot of the prepared test suspension was added to the disinfectant and mixed by vortexing. For the tests involving glutaraldehyde, sampling times were $2,4,8$, and 16 minutes for the $2.5 \%$ freshly activated solution and $4,8,16$, and 32 minutes for the $1.5 \%$ minimum effective concentration solution. In each case, $2.0-\mathrm{mL}$ samples were taken and added to $2.0 \mathrm{~mL}$ of a freshly prepared sodium bisulfite neutralizer. The concentration of sodium bisulfite was 2.2 times the assayed concentration of glutaraldehyde. For all tests involving OPA, 1-mL samples were taken at $1,2,4,8$, and 16 minutes for the $0.05 \%$ solution and at $0.5,1,2,4$, and 8 minutes for the $0.21 \%$ solution. Samples were added to 9-mL tubes of Letheen broth containing $1 \%$ fresh sodium bisulfite (neutralizer solution). All remaining procedures in the testing of both disinfectants were identical. After approximately 1 minute, serial dilutions $(1: 10)$ of neutralized samples were carried out in physiological saline solution to a predetermined point (dependent upon expected counts for each sample).

Phenol control evaluations of each prepared test suspension were performed as specified in the EPA test protocol. Tubes containing $9 \mathrm{~mL}$ of $0.8 \%$ phenol were equilibrated in a $25^{\circ} \mathrm{C}$ water bath and inoculated with $1 \mathrm{~mL}$ of test sus- 
pension. After 20 minutes, $1-\mathrm{mL}$ samples were taken and mixed with $9 \mathrm{~mL}$ of Letheen neutralizer solution. Serial dilutions (1:10) were carried out in physiological saline solution.

For all samples, selected dilution tubes (of neutralized suspensions) were assayed in duplicate for the number of viable organisms by membrane filtration. A $1-\mathrm{mL}$ aliquot of the chosen dilution was added to approximately $50 \mathrm{~mL}$ of physiological saline solution in a disposable plastic funnel (Microfil system, Millipore, Bedford, MA). Standard cellulose filters (Millipore, $0.45-\mu \mathrm{m}$ pore size, 47$\mathrm{mm}$ diameter) were used. After applying vacuum, filters were washed with an additional 50 to $100 \mathrm{~mL}$ of physiological saline solution and placed on Middlebrook 7H11 agar supplemented with 10\% BACTO-OADC enrichment (Difco, Detroit, MI). The agar plates were placed in 1-gallon polyethylene bags, sealed with twist ties, and incubated until colonies could be counted readily. This was approximately 20 to 24 days for both $M$ terrae and $M$ bovis, and 3 to 4 days for $M$ chelonae. While small colonies of $M$ terrae were counted after 14 days, plates were reincubated and assessed again at the same time as the $M$ bovis samples to ensure reliable colony counts. $M$ chelonae was incubated at $32^{\circ} \mathrm{C}$, whereas both $M$ terrae and $M$ bovis were incubated at $37^{\circ} \mathrm{C}$.

Results of the individual colony counts for the various assays are available on request.

\section{Disinfectant Neutralization Controls}

For all disinfectant samples, a neutralization verification was conducted with each organism on every test day. During the glutaraldehyde tests, this procedure consisted of inoculating a mixture of $2 \mathrm{~mL}$ of disinfectant and $2 \mathrm{~mL}$ of neutralizer with 400 organisms in $40 \mu \mathrm{L}$. In the OPA tests, $100 \mu \mathrm{L}$ containing 1,000 organisms was added to a mixture of $1 \mathrm{~mL}$ of disinfectant and $9 \mathrm{~mL}$ of neutralizer. This resulted in an inoculum of approximately $100 \mathrm{CFU} / \mathrm{mL}$ in each tube. In all cases, these suspensions were allowed to sit for at least 20 minutes prior to assay to detect any residual killing activity from unneutralized disinfectant. They then were assayed for viable organisms in a manner identical to the procedure described above. The percent inhibition for each control was determined as follows: percent inhibition $(\mathrm{PI})=[($ Expected \# of Colonies-Observed \# of Colonies)/(Expected \# of Colonies) $] \times 100$. The Expected \# of Colonies was obtained from the viable count assay for each test suspension.

\section{Statistical Methods}

Data for this study were collected over a total of 12 test days, 4 for glutaraldehyde, and 8 for OPA. Data for each disinfectant were analyzed separately. The response variable for both analyses was the time to a $6-\log _{10}$ reduction for each test organism. The times were calculated for each replication of every organism-disinfectant combination by linear regression of $\log \left(\mathrm{S} / \mathrm{S}_{0}\right)$ versus time, where $\mathrm{S}_{0}$ is the baseline count and $\mathrm{S}$ is the concentration of viable organisms at a particular sample time. The SAS (SAS Institute, Cary, NC) procedure REG was used for these regressions.

The 4 days of glutaraldehyde testing consisted of two suspensions of $M$ bovis (a and b, each prepared in different laboratories) exposed to two concentrations of glutaraldehyde (1.5\% minimum effective concentration and 2.5\% [fresh] solution). On every test day, each $M$ bovis suspension was tested against both concentrations of glutaraldehyde. Six- $\log _{10}$-reduction times were analyzed using a mixed-effects analysis of variance model. "Technician" and "test day" were deemed random factors, whereas "suspension" and disinfectant "concentration" were considered to be fixed factors. The variance components associated with the random factors were estimated using restricted maximum likelihood and were tested using an approximate $Z$ test. The main effects and the interaction of the fixed factors were evaluated with F statistics. All calculations were done using the MIXED procedure of SAS.

The 8 days of testing with OPA originally were designed as a balanced two-factor split-unit experiment, with days as whole units. The whole-unit factor was disinfectant "concentration" ( $0.05 \%$ and $0.21 \%)$ and the sub-unit factor was "organism" ( $M$ chelonae, $M$ terrae, and $M$ bovis). Concentrations were assigned to days, and every organism was tested on every day. Thus, every organism-concentration combination was replicated four times. As in the glutaraldehyde testing, two different suspensions of $M$ bovis ( $\mathrm{a}$ and $\mathrm{b}$ ) were used. This time, one was used for the first 4 days and the other for the last 4 days. In this analysis, it was decided to treat these suspensions as different organisms. Therefore, "organism" was partially a whole-unit factor and partially a split-unit factor. Due to variance heterogeneity, the $6-\log _{10}$ reduction times for each organism-concentration combination were transformed to the logarithmic scale for analysis. Because the design was an unbalanced split-unit design, statistical analysis was done using a maximum likelihood analysis of variance procedure. The SAS procedure MIXED was used for the analysis. "Test day" was treated as a random factor, whereas "organism," "concentration," and their interaction were treated as fixed factors. Adjusted means, along with their standard errors, were calculated for all organism-concentration combinations.

These were converted back to the original scale for presentation in figures and tables. Results expressed as time to achieve a $6-\log _{10}$ reduction are those determined by the described statistical regression procedures and do not necessarily represent an actual measurement at that point. Linear contrasts were used to investigate prespecified comparisons of means, such as the mean for $M$ bovis versus the mean for $M$ terrae at each of the disinfectant concentrations. $P$ values were adjusted for the multiplicity of contrasts examined.

\section{RESULTS}

Figure 1 shows the mean times (in minutes) required for a $6-\log _{10}$ reduction for each suspension of $M$ bovis tested against both concentrations of glutaraldehyde. Table 1 shows the results of the statistical analysis of these data. The $M$ bovis "a" suspension was significantly more resistant, rendering longer $6-\log _{10}$ reduction times across both glutaraldehyde concentrations. As expected, there was a significant difference between disinfectant concen- 


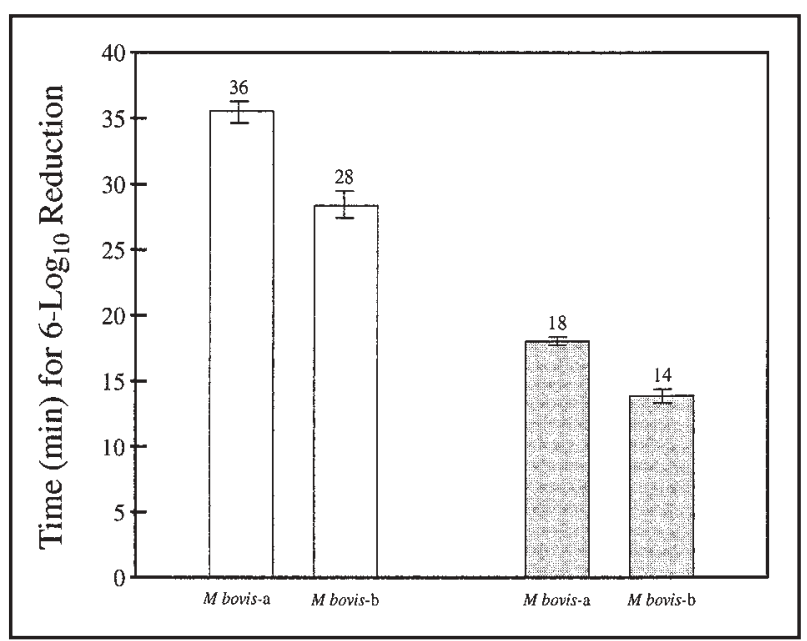

FIGURE 1. Mean times required to effect a $6-\log _{10}$ reduction of Mycobacterium bovis suspensions from two different laboratories ( $M$ bovis-a and $M$ bovis-b). Two glutaraldehyde concentrations were tested: $2.5 \%$ fresh solution (grey bars) and $1.5 \%$ minimum effective concentration (white bars). The precise result is shown above each column. The bars show the standard error of the mean.

TABLE 1

Statistical Analysis of Glutaraldehyde Data

\begin{tabular}{|c|c|c|c|c|}
\hline \multicolumn{5}{|c|}{ Variance Components Estimates* } \\
\hline Parameter & \multicolumn{2}{|c|}{ Estimate } & & \\
\hline Technician & \multicolumn{2}{|c|}{0.00} & & \\
\hline Test day & \multicolumn{2}{|c|}{2.87} & & \\
\hline Error & \multicolumn{2}{|c|}{5.58} & & \\
\hline \multicolumn{5}{|c|}{ Tests of Fixed Effects } \\
\hline & \multicolumn{2}{|c|}{ Degrees of Freedom } & \multirow[b]{2}{*}{ F Statistic } & \multirow[b]{2}{*}{$P>F$} \\
\hline Source & Numerator & Denominator & & \\
\hline Suspension $^{\dagger}$ & 1 & 8 & 22.9 & .001 \\
\hline Concentration ${ }^{\ddagger}$ & 1 & 8 & 183 & $<.001$ \\
\hline Interaction & 1 & 8 & 1.61 & .240 \\
\hline \multicolumn{5}{|c|}{$\begin{array}{l}\text { * Restricted maximum likelihood. } \\
\uparrow \text { Suspension A or suspension B. } \\
\neq 1.5 \% \text { (minimum effective) or } 2.5 \% \text { (fresh). }\end{array}$} \\
\hline
\end{tabular}

trations. The $1.5 \%$ minimum effective concentration produced times approximately twice those of the $2.5 \%$ fresh solution, across both $M$ bovis suspensions. Variance components for "technician" and "test day" were both small. The "suspension" by "concentration" interaction was nonsignificant, indicating that the effect of one factor on the 6$\log _{10}$ reduction times was not affected by a change in the other factor. In other words, both $M$ bovis suspensions gave the same results relative to each of the two concentrations of glutaraldehyde.

Figure 2 shows the mean times (in minutes) required by each concentration of OPA to effect a $6-\log _{10}$ reduction of each of the three Mycobacterium species involved in this study. Table 2 shows the statistical analysis of these data. The variance component for "test day" was small. There was a significant difference between test

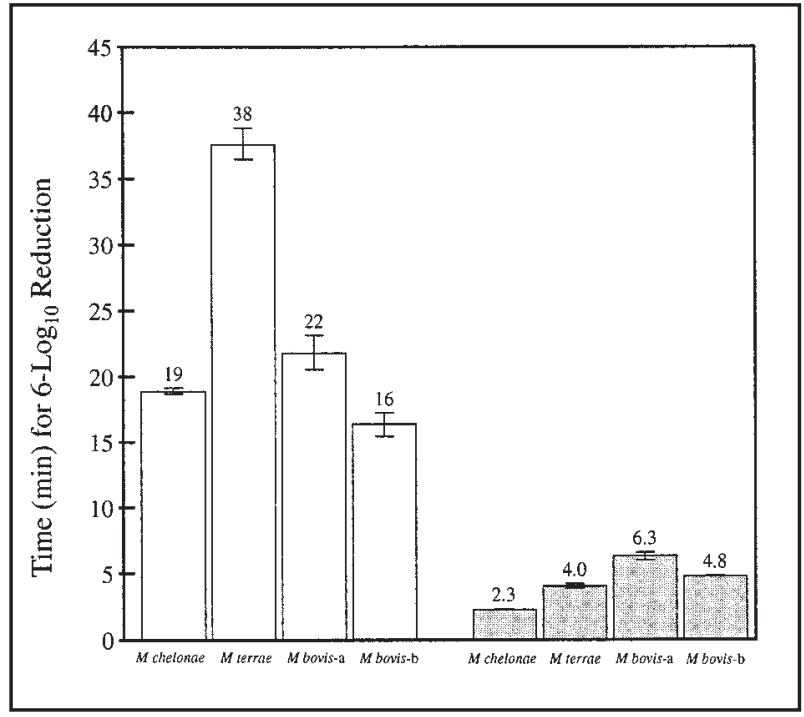

FIGURE 2. Mean times required to effect a $6-\log _{10}$ reduction for Mycobacterium chelonae, Mycobacterium terrae, and Mycobacterium bovis, using two different ortho-phthalaldehyde concentrations: $0.21 \%$ minimum effective concentration (grey bars) and $0.05 \%$ (white bars). $M$ bovis suspensions from two different laboratories ( $M$ bovis-a and $M$ bovis-b) were used. The precise result is shown above each column. The bars show the standard error of the mean.

TABLE 2

Statistical Analysis of Ortho-Phthalaldehyde Data

\begin{tabular}{|c|c|c|c|c|}
\hline \multicolumn{5}{|c|}{ Variance Components Estimates* } \\
\hline Parameter & & Estimate & & \\
\hline Test day & & 0.003 & & \\
\hline Error & & 0.010 & & \\
\hline \multicolumn{5}{|c|}{ Tests of Fixed Effects } \\
\hline & \multicolumn{2}{|c|}{ Degrees of Freedom } & \multirow[b]{2}{*}{ F Statistic } & \multirow[b]{2}{*}{$P>F$} \\
\hline Source & Numerator & Denominator & & \\
\hline Organism $^{\dagger}$ & 3 & 10 & 54.4 & $<.001$ \\
\hline Concentration ${ }^{\ddagger}$ & 1 & 6 & 869 & $<.001$ \\
\hline Interaction & 1 & 10 & 37.9 & $<.001$ \\
\hline
\end{tabular}

* Restricted maximum likelihood.

$\uparrow$ Mycobacterium bovis (A or B), Mycobacterium terrae, and Mycobacterium chelonae. $\doteqdot 0.21 \%$ (minimum effective) or $0.05 \%$.

“organisms” used. Disinfectant "concentrations” also differed significantly in activity, as expected. The "organism" by "concentration" interaction was also significant, indicating that the different species responded differently from one disinfectant concentration to the other. To examine these differences in more detail, specific contrasts were performed. These contrasts are detailed in Table 3 and, together with Figure 2, show the following:

1. Both $M$ bovis suspensions ( $\mathrm{a}$ and $\mathrm{b}$ ) react in a similar manner to changes in OPA concentration (Table 3 , line 1 ).

2. $M$ bovis and $M$ chelonae do not react in a similar way to changes in OPA concentration (Table 3 , line 2).

3. $M$ bovis and $M$ terrae do not react in a similar way to changes in OPA concentration (Table 3 , line 3 ). 
TABLE 3

Detailed Statistical Analysis of Ortho-Phthalaldehyde Data: Selected Individual Contrasts and Their Corresponding SigNIFICANCE

\begin{tabular}{|c|c|c|c|}
\hline Parameter & Estimate & SE & $\boldsymbol{P}$ \\
\hline \multicolumn{4}{|l|}{ Interactions with OPA concentrations } \\
\hline 1. Mycobacterium bovis: a vs b & 0.050 & 0.157 & .755 \\
\hline 2. $M$ bovis vs Mycobacterium chelonae & 0.872 & 0.102 & $<.001$ \\
\hline 3. $M$ bovis vs Mycobacterium terrae & 1.00 & 0.102 & $<.001$ \\
\hline 4. $M$ chelonae vs $M$ terrae & -0.130 & 0.102 & .232 \\
\hline \multicolumn{4}{|l|}{ Comparisons over OPA concentrations } \\
\hline 5. $M$ bovis: a vs b & -0.294 & 0.078 & .004 \\
\hline 6. $M$ chelonae vs $M$ terrae & -0.611 & 0.051 & $<.001$ \\
\hline \multicolumn{4}{|l|}{$0.05 \% \mathrm{OPA}$} \\
\hline 7. $M$ bovis vs $M$ terrae & 0.693 & 0.072 & $<.001$ \\
\hline 8. $M$ bovis-a vs $M$ terrae & 0.559 & 0.091 & $<.001$ \\
\hline 9. $M$ bovis-b vs $M$ terrae & 0.827 & 0.091 & $<.001$ \\
\hline \multicolumn{4}{|l|}{$0.21 \% \mathrm{OPA}$} \\
\hline 10. $M$ bovis vs $M$ terrae & -0.309 & 0.072 & .002 \\
\hline 11. $M$ bovis-a vs $M$ terrae & -0.468 & 0.091 & $<.001$ \\
\hline 12. $M$ bovis-b vs $M$ terrae & -0.150 & 0.091 & .131 \\
\hline
\end{tabular}

4. $M$ chelonae and $M$ terrae react in a similar way to changes in OPA concentration (Table 3 , line 4).

5 . When both concentrations of OPA are considered, there is a significant difference in the resistances of the two $M$ bovis suspensions (Table 3 , line 5).

6. When both concentrations of OPA are considered, $M$ terrae is significantly more resistant than $M$ chelonae (Table 3, line 6).

7. $M$ terrae is significantly more resistant to $0.05 \%$ OPA than either suspension of $M$ bovis (Table 3, lines 7-9).

8. $M$ terrae is significantly more susceptible to $0.21 \%$ OPA than $M$ bovis-"a," but not significantly different than $M$ bovis-"b" (Table 3, lines 10-12).

On every test day, each organism suspension was exposed to $0.8 \%$ phenol, as specified in the EPA protocol. $\log _{10}$ reductions were calculated for every exposure, and, after completing all test days, mean values were determined for each organism. As shown in Figure 3, both $M$ chelonae and $M$ terrae were significantly more resistant to $0.8 \%$ phenol than was $M$ bovis.

An analysis of the percent inhibition data to confirm neutralization efficacy indicated that all disinfectants were neutralized completely following their specific contact period (data not shown).

\section{DISCUSSION}

The use of a standardized mycobacterial test suspension is an issue central to effective and meaningful tuberculocidal testing. Although $M$ bovis has been the standard for tuberculocidal testing in the United States, other species may prove ultimately to be more suitable. Rapidgrowing, less virulent saprophytic species of mycobacteria, such as $M$ smegmatis, have been considered for tubercu- locidal testing in the past, but it has since been demonstrated that such species are more susceptible to disinfection than $M$ tuberculosis. ${ }^{18-21} M$ chelonae was included in the present study, to evaluate the performance of a representative rapid-growing species against OPA. As expected, this organism demonstrated significantly less resistance to OPA than either of the other species tested. Clearly, estimates of tuberculocidal activity based on data from such rapid-growing species are not predictive of activity against either $M$ bovis or clinical tuberculosis isolates. ${ }^{14}$

$M$ terrae, an intermediate grower, may represent a more reasonable alternative. This study showed $M$ terrae to be either similar or more resistant to OPA than $M$ bovis. Although relatively unexplored in the United States, European researchers have shown this organism to have resistance comparable to, or greater than, $M$ tuberculosis. In 1978, Sonntag conducted a comparative analysis of $M$ terrae and $M$ tuberculosis against several disinfectants. ${ }^{22}$ It was found that the two species exhibited approximately equal resistance to aldehydes, phenols, amphotensides, and peracetic acid. Seven years later, Sonntag and Hingst presented a comparison of $M$ terrae and $M$ tuberculosis using six different disinfectants. ${ }^{23}$ Their findings again revealed very similar resistance profiles between the two organisms, this time with $M$ terrae showing slightly greater resistance to all of the disinfectants tested. Also of possible significance was their finding of high levels of common membrane antigens shared by these two species. Similar results were achieved by vanKlingeren and Pullen. ${ }^{24}$ After finding $M$ terrae and $M$ tuberculosis to have similar resistance to 11 disinfectants, they recommended the use of $M$ terrae for quantitative suspension tuberculocidal testing. A 1998 study by Griffiths et al provided recent confirmation 
of these findings. ${ }^{25}$ They reported $M$ terrae either to equal or exceed $M$ tuberculosis in resistance to six disinfectants. In addition, the German Society for Hygiene and Microbiology already has declared $M$ terrae its official tuberculocidal test species. ${ }^{26}$

Obviously, the most important consideration when selecting an organism to assess tuberculocidal activity is the way it behaves in relation to $M$ tuberculosis or a similar documented standard. This study involved a comparative analysis of $M$ chelonae and $M$ terrae against the current EPAapproved test organism, $M$ bovis. Previous work done in our laboratory has shown that standard $M$ bovis suspensions often differ from laboratory to laboratory. ${ }^{7}$ For this reason, two different $M$ bovis suspensions (each prepared in different laboratories) were used. Not surprisingly, the two suspensions gave slightly different results. Nonetheless, $M$ terrae showed similar resistance to $M$ bovis when subjected to a new, highly effective chemical disinfectant not evaluated in any of the aforementioned studies. These results support the conclusions of other researchers who have proposed using $M$ terrae in tuberculocidal testing of aldehydes. Along with possessing a resistance profile similar to $M$ tuberculosis (and in this case, $M$ bovis), the lower virulence and more rapid growth of $M$ terrae make it an attractive alternative to the current $M$ bovis standard. Interestingly, the resistances of $M$ bovis, $M$ chelonae, and $M$ terrae to the $0.8 \%$ phenol standard were very different (Figure 3 ). $M$ chelonae, although the organism most sensitive to OPA, was by far the most resistant to phenol. These results raise old doubts concerning the validity of the phenol resistance window that all $M$ bovis suspensions used for tuberculocidal testing currently are required to meet. They reinforce the fact that the phenol resistance of a particular organism does not necessarily correlate with its resistance to other disinfectants.

The rapid tuberculocidal activity of OPA is a separate important finding of this study. Although glutaraldehyde has long been the standard for high-level disinfection among chemical germicides, its tuberculocidal activity has been documented to be relatively slow. In a 1995 analysis of three glutaraldehyde-based disinfectants, Jette et al concluded that "the search for a safer, non-damaging and 'userfriendly' alternative to glutaraldehyde should continue as safer more dilute solutions require long, impractical exposure times for high level disinfection." 27 A comparison of the tuberculocidal activities of glutaraldehyde and OPA can be made by contrasting Figures 1 and 2 . At the minimum effective concentration of $1.5 \%$, glutaraldehyde required a mean of 32 minutes to achieve a $6-\log _{10}$ reduction of $M$ bovis. In contrast, OPA at its minimum effective concentration of $0.21 \%$ required a mean of only 5.5 minutes to achieve the same level of kill, making OPA nearly six times faster than alkaline glutaraldehyde by this measure.

OPA has been shown to have other advantages over glutaraldehyde as a hospital-level disinfectant. In 1993, Alfa and Sitter conducted a thorough in-hospital assessment of the effectiveness of $0.5 \%$ OPA on bronchoscopes, gastroscopes, and colonoscopes. ${ }^{28}$ Instruments were checked for the presence of residual bacteria, viruses, parasites, and

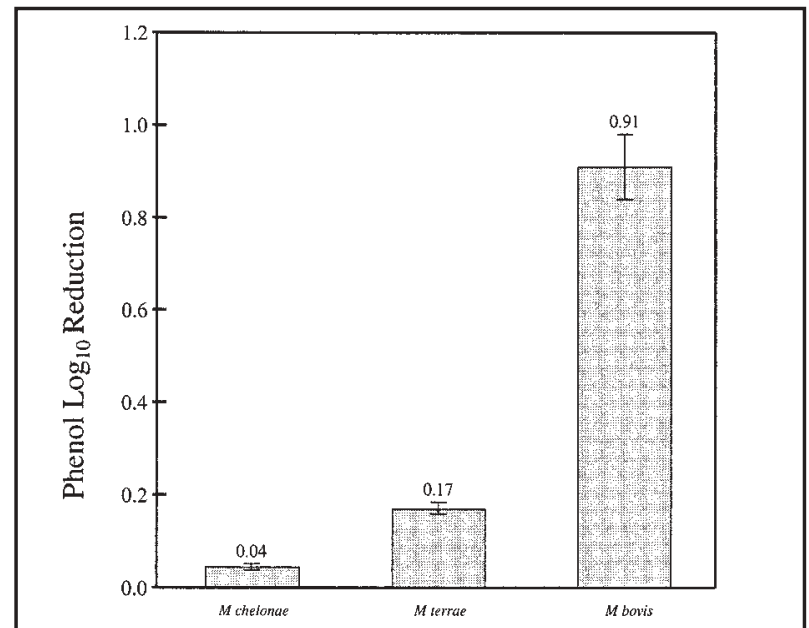

FIGURE 3. Mean $\log _{10}$ reductions for Mycobacterium chelonae, Mycobacterium terrae, and Mycobacterium bovis produced by exposure to $0.8 \%$ phenol at $25^{\circ} \mathrm{C}$ for 20 minutes. The precise result is shown above each column. The bars show the standard error of the mean.

fungi after a 5-minute exposure to OPA at room temperature. Of the 100 endoscopes disinfected following use, none of these organisms could be detected (the detection limit being $10 \mathrm{CFU} / \mathrm{mL}$ ). Data from microbial load assays prior to disinfection indicated that OPA effected greater than a $5-\log _{10}$ reduction of organisms (protected by extensive organic material) within 5 minutes. They further confirmed that the concentration of OPA remained $>0.45 \%$ over the entire 14 day test period for all 19 batches tested. These data are consistent with a study by Robison et al in which both $0.5 \%$ OPA and $2 \%$ alkaline glutaraldehyde were compared in a clinicaluse crossover study involving four dental operatories. ${ }^{29}$ This study compared the antimicrobial and clinical characteristics of both disinfectants. OPA was found to have a significantly higher antimicrobial activity than glutaraldehyde against $M$ bovis, polio virus, and Candida albicans under all phases of clinical use. The mean tuberculocidal times of the $0.5 \%$ OPA were roughly one tenth those of the glutaraldehyde. Even after 3 weeks of storage and loading of the OPA solution with $10 \%$ human whole blood, its tuberculocidal activity remained at least two times faster than that of the fresh glutaraldehyde solution. Because OPA does not polymerize, the concentration of active ingredient did not decrease with age alone. Also, consistent with the results of Alfa and Sitter, the detrimental effects of dilution caused by clinical use were minimal for the OPA solutions. Clinically, OPA was preferred by most users because it was less corrosive than glutaraldehyde and had a much less objectionable odor.

In summary, this study has shown that $M$ terrae and $M$ bovis have similar resistances to OPA. The rapid grower, $M$ chelonae, was significantly more susceptible. This, viewed with similar studies using other disinfectants, lends support to the consideration of $M$ terrae as a replacement for $M$ bovis in tuberculocidal testing of aldehydes. In addition, this work confirms the efficacy and celerity of OPA as a tuberculocidal disinfectant, acting with nearly six times 
the speed of glutaraldehyde at their respective minimum effective concentrations.

\section{REFERENCES}

1. Alvarado CJ, Stolz SM, Maki DG. Nosocomial infection and pseudoinfection from contaminated endoscopes and bronchoscopes. MMWR 1991;40:675-678 (including editorial note).

2. Nelson KE, Larson PA, Schraufnagel DE, Jackson J. Transmission of tuberculosis by flexible fiberbronchoscopes. Am Rev Respir Dis 1983;127:97-100

3. Spach DH, Silverstein FE, Stamm WE. Transmission of infections by gastrointestinal endoscopy and bronchoscopy. Ann Intern Med 1993;118:117-128.

4. Wheeler PW, Lancaster D, Kaiser AB. Bronchopulmonary cross-colonization and infection related to mycobacterial contamination of suction valves of bronchoscopes. J Infect Dis 1989;159:954-958.

5. Ascenzi JM, Ezzell RJ, Wendt TM. A more accurate method for measurement of tuberculocidal activity of disinfectants. Appl Environ Microbiol 1987;53:2189-2192.

6. Isenberg HD, Giugliano ER, France K, Alperstein P. Evaluation of three disinfectants after in-use stress. J Hosp Infect 1988;11:278-285.

7. Robison RA, Osguthorpe RJ, Carroll SJ, Leavitt RW, Schaalje GB, Ascenzi JM. Culture variability associated with the US Environmental Protection Agency Tuberculocidal Activity Test Method. Appl Environ Microbiol 1996;62:2681-2686.

8. Newman KA, Tenney JH, Oken HA, Moody MR, Wharton R, Schimpff SC. Persistent isolation of an unusual Pseudomonas species from a phenolic disinfectant system. Infect Control 1984;5:219-222.

9. Sautter RL, Mattman LH, Legaspe RC. Serratia marcescens meningitis associated with contaminated benzalkonium chloride solution. Infect Control 1984;5:223-225.

10. vanKlingeren B, Pullen W. Glutaraldehyde resistant mycobacteria from endoscope washers. J Hosp Infect 1993;25:147-149.

11. Russell AD, Hammond SA, Morgan JR. Bacterial resistance to antiseptics and disinfectants. J Hosp Infect 1986;7:213-225.

12. US Environmental Protection Agency. Tuberculocidal activity test method. In: Campt DD, ed. Data Call-In Notice for Tuberculocidal Effectiveness Data for All Antimicrobial Pesticides With Tuberculocidal Claims. Washington, DC: US Environmental Protection Agency; 1988:1-8.

13. Association of Official Analytical Chemists. Tuberculocidal activity of disinfectants. In: Williams S, ed. Official Methods of Analysis of the AOAC. 14th ed. Arlington, VA: AOAC; 1984:73-74.

14. Rutala WA, Cole EC, Wannamaker NS, Weber DJ. Inactivation of
Mycobacterium tuberculosis and Mycobacterium bovis by 14 hospital disinfectants. Am J Med 1991;91(suppl 3B):267S-270S.

15. Collins FM, Montalbine V. Mycobactericidal activity of glutaraldehyde solutions. J Clin Microbiol 1976;4:408-412.

16. Cole EC, Rutala WA, Nessen L, Wannamaker NS, Weber DJ. Effect of methodology, dilution, and exposure time on the tuberculocidal activity of glutaraldehyde-based disinfectants. Appl Environ Microbiol 1990;56:1813-1817.

17. Urayama S, Kozarek RA, Sumida S, Raltz S, Merriam L, Pethigal P. Mycobacteria and glutaraldehyde: is high-level disinfection of endoscopes possible? Gastrointest Endosc 1996;43:451-456.

18. Association of Official Analytical Chemists. Tuberculocidal activity of disinfectants. In: Official Methods of Analysis of the AOAC. 13th ed. Arlington, VA: AOAC; 1980:65-66.

19. Association Francaise de Normalisation (AFNOR). Recueil de normes francaises des antiseptiques et desinfectants. In: Antiseptics and Disinfectants. Edition bilingue. Paris, France: AFNOR; 1981.

20. Best M, Sattar SA, Springthorpe VS, Kennedy ME. Comparative mycobactericidal efficacy of chemical disinfectants in suspension and carrier tests. Appl Environ Microbiol 1988;54:2856-2858.

21. Best M, Sattar SA, Springthorpe VS, Kennedy ME. Efficacies of selected disinfectants against Mycobacterium tuberculosis. J Clin Microbiol 1990;28:2234-2239.

22. Sonntag HG. Desinfektions verfahren bei Tuberculose. Hygiene und Medizin 1978;3:322-325.

23. Sonntag HG, Hingst V. Comparative studies on the effects of disinfectants on $M$. tuberculosis and $M$. terrae. Zentrablatt Bakteriologie B: Hygiene 1985;181(Serie B):31. Abstract.

24. vanKlingeren $\mathrm{B}$, Pullen W. Comparative testing of disinfectants against Mycobacterium tuberculosis and Mycobacterium terrae in a quantitative suspension test. J Hosp Infect 1987;10:292-298.

25. Griffiths PA, Babb JR, Fraise AP. Mycobacterium terrae: a potential surrogate for Mycobacterium tuberculosis in a standard disinfectant test. $J$ Hosp Infect 1998;38:183-192.

26. Hingst V, Wurster C, Sonntag HG. A quantitative test method for the examination of antimycobacterial disinfection procedures. Zentrablatt fur Hygiene und Umweltmedizin 1990;190:127-140.

27. Jette LP, Ringuette L, Ishak M, Miller M, Saint-Antoine P. Evaluation of three glutaraldehyde-based disinfectants used in endoscopy. J Hosp Infect 1995;30:295-303.

28. Alfa MJ, Sitter DL. Inhospital evaluation of orthophthalaldehyde as a high level disinfectant for flexible endoscopes. J Hosp Infect 1994;26:15-26.

29. Robison RA, Robinson DF, Ploeger BJ, Christensen RP. Clinical and laboratory efficacy tests of a new disinfectant. J Dent Res 1991;70:438. Abstract. 